active monitoring (regular assessment of disease status) drank more fruit/vegetable juice than men who had received surgery $(p=0.004)$. Conclusion Diagnosis of localised PC prompted around one third of men to adopt healthier diets and eat more 'prostate-healthy' foods. Dietary choices were influenced by treatment received, with more interest in dietary factors for survivorship in men undergoing active monitoring than those undergoing radical treatment. PC survivors are motivated to improve their diet and are able to access and act on healthy eating advice. Diagnosis of PC may serve as a "teachable moment", thereby providing opportunities for clinicians to provide targeted advice that could be beneficial to general (and prostate-specific) health and to support survivorship.

\section{PS06 FINDING THE MISSING UNITS: IDENTIFYING UNDER- REPORTING OF ALCOHOL CONSUMPTION IN ENGLAND}

doi:10.1136/jech-2012-201753.105

S Boniface. Epidemiology and Public Health, UCL, London, UK

Background Alcohol sales coverage (reported consumption as a percentage of total sales) is typically $40-60 \%$. This study explores how accidental under-estimation of alcohol in drinks poured at home might contribute to low sales coverage.

Methods 283 drinking adults (16+) completed a face-to-face survey comprising a questionnaire and pouring task on 12 occasions at six sites in and near London in summer 2011. Actual and perceived number of units poured in a 'usual glass' of wines and spirits is explored by demographic and social factors.

Results There were 283 participants (54\% women) who completed the questionnaire and pouring task. The 283 participants poured a total of 465 glasses of wine and spirits. $52 \%$ wine estimates and $42 \%$ spirit estimates were within 0.5 units of their actual value. More participants over-estimated the amount of alcohol they had poured than under-estimated. Multinomial logistic regression found significant risk factors for under-estimating wine were increasing volume of wine poured (RRR $1.0295 \%$ CI 1.01-1.02, $\mathrm{P}<0.0001$ ), belonging to a non-white ethnicity $(\mathrm{RRR} 3.88,1.65-9.16, \mathrm{P}=0.002)$, and being unemployed or retired ( $R R R$ 4.30, 1.08-17.07, $\mathrm{P}=0.038$ ). Only the volume of spirits poured was a significant predictor of under-estimating spirits ( $R R R$ 1.04, 1.01-1.06, $\mathrm{P}=0.003$ ). For both wines and spirits, not having a degree was significantly associated with an increased risk of over-estimating the amount of alcohol poured.

Conclusion As participants were more likely to over-estimate than under-estimate, this survey is not able to explain low sales coverage as a product of accidental under-estimation of the amount of alcohol poured drinks drunk at home. A future qualitative study will explore how recall accuracy may contribute to low sales coverage.

\section{PS07 TRENDS IN BLO0D PRESSURE IN ENGLAND: G00D TREATMENT OR GOOD LUCK?}

doi:10.1136/jech-2012-201753.106

SJ DeWilde, IM Carey, SM Shah, T Harris, AR Rudnicka, PH Whincup, DG Cook. PHSE, St George's, University of London, London, UK

Background For 30 years, adult blood pressure (BP) has declined in many developed countries. This is likely to have contributed to declining cardiovascular disease mortality. However, the reasons for this, particularly the impact of increased antihypertensive treatment, have been little studied. Diagnostic criteria have changed, meaning that more patients are treated at lower levels of BP than previously; and treatment intensity is greater. Accounting for the effects of treatment on BP trends is complex and open to misinterpretation.

Methods Using the Health Survey for England we examined BP measures and treatment in white subjects aged 18+ years over two periods, 1994-2002 and 2003-2009. To examine trends independent of anti-hypertensive treatment, untreated BP was estimated from the recorded BP on treatment. To do this, a model was derived using published data on the effect of anti-hypertensives used singly and in combination at differing pre-treatment BP levels. BP untreated with statins was similarly estimated.

Results Among an average 9,147 subjects per year, mean systolic BP (SBP) in men declined by $0.55 \mathrm{mmHg}$ annually $(95 \%$ CI 0.46 to 0.64 ) between $1995-2002 ; 0.34 \mathrm{mmHg}$ annually (95\% CI 0.21 to 0.46 ) between 2003-2009. Similar trends occurred in women. The decline was greater in older groups, but present in young, largely untreated groups, although the trend was greatly reduced in young men by 2009. Trends in estimated SBP adjusting for the influence of treatment, where present, exhibited only slightly lower declines; for men: $0.47 \mathrm{mmHg}$ annually (95\% CI 0.37 to 0.57 ) between 1995 2002; $0.27 \mathrm{mmHg}$ annually (95\% CI 0.13 to 0.41 ) between 2003 2009. Statin therapy had little additional effect. Diastolic blood pressure showed similar but weaker trends.

Conclusion For 15 years, BP declined in English adults. The overall decline in SBP of nearly $5 \mathrm{mmHg}$ over the study period is likely to be of clinical significance in reducing CVD events. For an individual aged $40-49$ a $5 \mathrm{mmHg}$ reduction in SBP, as seen here, would be expected to reduce the risk of stroke by $23 \%$ and of IHD by $16 \%$. It is therefore of concern that, in recent years, the decline has essentially ceased in the youngest age groups, particularly in younger men. The effect of treatment was modest; less than $25 \%$ of the male SBP decline is attributable to it. Other explanations for this fall, occurring whilst obesity has increased, need further exploration, but reduced salt intake is a likely candidate.

\section{PSO8 IS THERE A PROTECTIVE EFFECT OF HIGH MINERAL CONTENT IN DRINKING WATER ON CORONARY HEART DISEASE MORTALITY? A CROSS-SECTIONAL ECOLOGICAL STUDY OF A LARGE POPULATION IN CENTRAL ENGLAND}

doi:10.1136/jech-2012-201753.107

GM Rudge, MA Mohammed. School of Health and Population Sciences, University of Birmingham, Birmingham, UK

Background It has been hypothesised that dissolved minerals in water, particularly calcium carbonate and magnesium carbonate, are protective against heart disease. Animal studies suggest biologically plausible mechanisms for this and statistically significant effects have been presented from several large ecological studies.

Setting The West Midlands Government Office Region in central England, has a large and diverse population which has great variation in water hardness over a relatively small area. Our units of analysis were West Midlands neighbourhoods (lower level super output areas) falling in areas supplied by two water companies. We obtained exposure estimates from water industry measurements taken in 2007.

Methods Using geographical information system software, digitised supply water supply maps were mapped to our neighbourhood geography. Having established the distribution of water hardness through neighbourhoods, we mapped tertiles of hardness and identified neighbourhoods inside them. To these we linked emergency myocardial infarction (MI) admissions for 45-74 year-old residents for a three year period, adjusting for the age distribution of the neighbourhoods, ethnic mix and socio-economic deprivation. We used a negative binomial model to determine the degree of association between water hardness and MI admission counts, adjusting for the other variables.

Results We were able to accurately determine the mineral content of water supplied to 2,925 neighbourhoods with a total population of over 4.5 million. Contrary to other studies, we found just a small but non-significant negative correlation between hardness and MI admissions were seen in men, with an incidence rate ratio (IRR) of 0.97 , per tertile, $(0.92-1.03, \mathrm{p}=0.37)$ and a small, non-significant positive one seen in women, IRR=1.02, $(0.93-1.12, p=2)$. 
Conclusion We found no evidence of a protective effect of hard water against acute MI admission, contrary to other published studies. This may be because our methods allowed us to better account for possible confounding variables. We go on to hypothesise that as water supply networks developed historically, with soft water from upland areas being used supply the needs of rapidly industrialising areas and other areas left to use local groundwater, some interesting accidental correlations between neighbourhood socio-economic status and water hardness arose which may have confounded previous work. However, mass differential exposure to highly bio-available minerals, especially calcium and magnesium, does raise some interesting further questions about the relationship between water supply and population health, which our methods can be used to investigate further.

\section{PSO9 SLEEP PROBLEMS: AN EMERGING GLOBAL EPIDEMIC? FINDINGS FROM THE INDEPTH WHO-SAGE STUDY AMONG OVER 40,000 OLDER ADULTS FROM EIGHT COUNTRIES ACROSS AFRICA AND ASIA}

doi:10.1136/jech-2012-201753.108

'S Stranges, 'W Tigbe, ${ }^{2} \mathrm{FX}$ Gómez-Olivé, 1'2M Thorogood, 'N-B Kandala. 'Division of Health Sciences, University of Warwick Medical School, Coventry, UK; ${ }^{2}$ MRC/Wits Rural Public Health \& Health Transitions Research Unit, University of the Witwatersrand, Agincourt, South Africa

Background Several studies have reported downward trends in the average duration of sleep and an increasingly higher prevalence of sleep problems across different Western populations. However, the evidence from low-income countries is limited. This study aims to fill this gap by examining the prevalence of sleep problems and associated factors in low-income settings.

Methods Community-wide samples were taken from eight countries across Africa and Asia participating in the INDEPTH WHOSAGE multicentre collaboration during 2006-2007. The participating sites included rural populations in Ghana, Tanzania, South Africa, India, Bangladesh, Vietnam and Indonesia, and an urban area in Kenya. The overall sample comprised 24,434 women and 19,501 men, for a total of 43,935 participants, aged 50 years and over. Two measures of sleep quality, over the last 30 days, were assessed alongside a number of socio-demographic variables, measures of quality of life, and co-morbidities.

Results Overall $16.6 \%$ of participants reported severe/extreme nocturnal sleep problems, with a striking variation across the eight populations, ranging from 3.9\% (Purworejo, Indonesia, and Nairobi, Kenya) to over $40.0 \%$ (Matlab, Bangladesh). There was a consistent pattern of higher prevalence of sleep problems in women and older age groups. In bivariate analyses, lower education, not living in partnership, and poorer self-rated quality of life were consistently associated with higher prevalence of sleep problems $(\mathrm{P}<0.001)$. In multivariate logistic regression analyses, limited physical functionality or greater disability and feelings of depression and anxiety were consistently strong, independent correlates of sleep problems, both in women and men, across the eight sites $(\mathrm{P}<0.001)$.

Conclusion A large number of older adults in low-income settings are currently experiencing sleep problems, which emphasises the global dimension of this emerging public health issue. This study corroborates the multifaceted nature of sleep problems, which are strongly linked to poorer general wellbeing and quality of life, and psychiatric co-morbidities.

\section{PS10 THE APPROPRIATENESS OF, AND COMPLIANCE WITH, TELEPHONE TRIAGE DECISIONS}

doi:10.1136/jech-2012-201753.109

L Blank, J Coster, A Cathain, E Knowles, J Tosh, J Turner, J Nicholl. ScHARR, University of Sheffield, Sheffield, UK
Background Telephone triage plays an important role in managing demand for healthcare. In recent years there has been an increasing use of telephone triage in managing demand for unscheduled health care for general health problems. Policy makers, service providers and service evaluators are interested in the appropriateness of triage decisions in the context of offering an effective and safe service. In 2009 the Department of Health in England announced the establishment of a small number of pilot sites to test a new telephone triage service called 'NHS 111'. The service offers telephone triage to members of the general population calling about urgent but nonemergency health problems. Calls are triaged by trained non-clinical call advisors and directed to a range of services such as an emergency department, or a general practitioner.

Methods As part of an evaluation of these pilots, in order to provide context for understanding the findings, a rapid evidence assessment was undertaken of the appropriateness of, and compliance with, telephone triage to synthesise the evidence on the percentage of telephone triage decisions assessed as appropriate and the percentage which callers complied with. The aim of this paper is to report on a systematic review of the literature on appropriateness of, and compliance with, telephone triage decisions. The study focused on telephone triage services directing patients to an appropriate health care provider. The principles of rapid evidence assessment were followed.

Results We identified 54 relevant papers; 26 papers reported appropriateness of triage decision, 26 papers reported compliance with triage decision and 2 papers reported both. Nurses triaged calls in most of the studies $(n=49)$. Triage decisions rated as appropriate varied between $44 \%$ and $98 \%$ (median 75 ); compliance ranged from $56 \%$ to $98 \%$ (median $77 \%$ ). Variation could not be explained by type of service or method of assessing appropriateness. However, inconsistent definitions of appropriateness may explain some variation. Triage decisions to contact primary care (median 66\%, range 25\%91\%) may have lower compliance than decisions to contact emergency services (median $75 \%$, range $29 \%-100 \%$ ) or self care (median $77 \%$, range $26 \%-100 \%$ )

Conclusion Telephone triage services can offer appropriate decisions, and decisions that callers comply with. However the association between the appropriateness of a decision and subsequent compliance requires further investigation and further consideration needs to be given to the minority of calls which are inappropriately managed. We suggest that a definition of appropriateness incorporating both accuracy and adequacy of triage decision should be encouraged.

\section{PS11 DOES CONTACT WITH A PODIATRIST PREVENT THE OCCURRENCE OF A LOWER EXTREMITY AMPUTATION IN PEOPLE WITH DIABETES? A SYSTEMATIC REVIEW AND META-ANALYSIS}

doi:10.1136/jech-2012-201753.110

${ }^{1} \mathrm{CM}$ Buckley, ${ }^{2} \mathrm{PM}$ Kearney, ${ }^{2} \mathrm{JJ}$ Perry, ${ }^{1} \mathrm{CP}$ Bradley. ${ }^{1}$ Department of GP, UCC, Cork, Ireland; ${ }^{2}$ Department of Epidemiology and Public Health, UCC, Cork, Ireland

Background Diabetes is associated with a significant risk of LEA (lower extremity amputation). LEA rates vary between communities, 46-9,600 per $10^{5}$ people with diabetes, for many reasons. The effects of clinical and socio-demographic risk factors on the occurrence of a LEA have been well documented in people with diabetes. However, the effect of patient contact with a podiatrist on the prevention of LEA in people with diabetes is less well explored. The objective of this study was to determine if contact with a podiatrist prevents the occurrence of lower extremity amputation in people with diabetes.

Methods We conducted a systematic review of available published literature on the effect of contact with a podiatrist on the prevention of lower extremity amputation in people with diabetes. Eligible 